Australasian Journal of Philosophy 79 (2001), pp. 90-106.

\title{
THE ROLE OF COHERENCE IN EPISTEMIC JUSTIFICATION
}

\section{Tomoji Shogenji}

Among many reasons for which contemporary philosophers take coherentism in epistemology seriously, the most important is probably the perceived inadequacy of alternative accounts, most notably misgivings about foundationalism. But coherentism also receives straightforward support from cases in which beliefs are apparently justified by their coherence. From the perspective of those against coherentism, this means that an explanation is needed as to why in these cases coherence apparently justifies beliefs. Curiously, this task has not been carried out in a serious way in the anti-coherentist literature although there is no scarcity of objections to coherentism. The traditional charge has been that justification by coherence is circular. More recently the isolation problem allegedly reveals that coherentism justifies beliefs that should not be justified. Questions have also been raised with respect to the basing relation and feasibility. ${ }^{1}$ However, these objections do not explain why some beliefs appear to be justified by their coherence. This paper fills this gap in the anti-coherentist literature by offering a noncoherentist account of justification by coherence.

The paper proceeds as follows. Section I delineates the framework of discussion and develops some conceptual tools needed in later analyses. Section II argues that there are genuine cases of an increase in existing empirical justification by coherence, but that it does not require coherence to generate additional justification - coherence serves as a channel of justification among beliefs, which is no more problematic than channeling of justification from basic to derived beliefs in foundationalism. Section III makes a stronger case for justification by coherence, where each of the coherent beliefs has no independent empirical justification; but Section IV argues that even in these cases coherence need not generate justification - coherence licenses the channeling of justification from outside sources. The upshot is that although these are cases in which coherence helps justification of beliefs, coherence need not generate justification. Instead coherence either serves as a channel of justification or licenses the channeling of justification. Thus, justification by coherence in these cases must be embedded in a noncoherentist theory of epistemic justification, such as foundationalism, which is to explain how justification is generated in the first place.

I. Preliminaries 
This section delineates the framework of discussion and develops some conceptual tools. I begin with remarks on the scope of our investigation. First, our subject of investigation is epistemic justification of beliefs, as opposed to their pragmatic justification. We may at times be justified in believing some propositions despite the contrary evidence because the act of believing them leads to desirable consequences, ${ }^{2}$ or perhaps it is morally the right thing to believe. This paper does not address such non-epistemic justification of beliefs. I will take beliefs to be justified only if something indicates their truth. Related to this focus on truth-conduciveness is the internalist conception of justification assumed throughout this paper. The main business of epistemology in my view is to explore ways of increasing true beliefs and reducing false beliefs by utilizing resources available to us. Information inaccessible to the epistemic agent is irrelevant to this agent-centered teleological project in epistemology, i.e., the project of finding principles that help the epistemic agent in acquiring and retaining more truth beliefs and less false beliefs. ${ }^{3}$

This does not mean that I reject externalism — whose characterizations of epistemic concepts, such as knowledge and justification, refer to information inaccessible to the epistemic agent-as an analysis of these concepts as they are employed by certain people (e.g., the native speakers of contemporary English). If externalism turns out to be the correct analysis of the way some people use certain concepts, we should conclude that these people do not use them (solely) for the purpose of increasing true beliefs and reducing false beliefs by utilizing resources available to them. We had better stay away then from such concepts in our agent-centered teleological project lest we should go astray because of the extraneous elements in them. ${ }^{4}$ In making this suggestion, I am not saying that people's intuitive judgment as to whether the agent has knowledge, justification, etc., in particular cases (according to their understandings of these epistemic concepts) is irrelevant to our inquiry. It is relevant if the intuition is based on information available to the epistemic agent. But even then, intuition should be treated as a (very) fallible guide - we often make mistakes in our intuitive epistemic judgment (Kahneman, Slovic and Tversky [9]). We cannot settle important problems of epistemology by epistemic intuition.

Second, our subject of investigation is empirical justification, as opposed to a priori justification. We may at times be justified in believing some propositions independently of any empirical evidence and solely because of their propositional contents, most notably in logic and mathematics. But this paper only discusses empirical justification, taking no position on the nature and extent of a priori justification. When I need to take a side on some issues of a priori justification to evaluate the viability of an epistemological option, I will adopt whatever position that favors coherentism in empirical justification. This should ensure that my critical analyses of coherentism in empirical justification depend on no assumptions against it on the nature and extent of a priori justification.

Third, our subject of investigation is justifiability of beliefs as opposed to the justification of the agent in her beliefs. It is widely acknowledged among epistemologists that even if a certain proposition is justifiable by the evidence available to the agent, she may not be justified in believing the proposition because she believes it for the wrong reason, and not on the basis of the relevant evidence she has. This paper does not address this issue of basing, which is needed for the full analysis of doxastic justification. In what 
follows I will regard a belief as justified provided the agent has appropriate evidence for it, assuming that she acquires and retains her belief on the basis of the relevant evidence. ${ }^{5}$

The next set of remarks concerns the probabilistic conception of justification. Throughout this paper I take empirical justification to be a matter of degree. This means first of all that I make no demand that a justified belief be infallible, and further that I set up no threshold of probability for justification. In other words, justification comes in various degrees and there is no particular point in the scale of probability at which a belief changes its status from being unjustified to being justified. Consequently, when I ask whether beliefs are justified by their coherence, the issue is whether they are more justified because of their coherence. This should be much easier to establish than showing their high degrees of probability beyond some threshold or guaranteeing their truth with absolute certainty. The probabilistic approach also allows a sensible characterization of the concept of coherence. It is commonly held that coherence of beliefs requires mutual support among them. If one insists that a belief supports only those beliefs that follow from it infallibly, there are hardly any non-trivial cases of mutual support among our actual beliefs. It is much easier to find cases of probabilistic mutual support because people often hold a set of beliefs each member of which is rendered more likely to be true by the remainder of the set. ${ }^{6}$

These ramifications of the probabilistic approach are fairly obvious, but some implications of probabilification seem to be still underappreciated by epistemologists. One of them is the reciprocity of the probabilistic support between two propositionsi.e., any non-trivial probabilistic support between two propositions is mutual, and thus proposition A supports proposition B if and only if they are coherent (mutually supportive). ${ }^{7}$ This is because the following principle of the Reciprocity of Probabilistic Support (RPS for short) is a theorem in the probability calculus. ${ }^{8}$

RPS: For any propositions $A$ and $B$ such that $\operatorname{Prob}(A) \neq 0$ and $\operatorname{Prob}(B) \neq 0, A$ supports $B$ if and only if $B$ supports $A$ [i.e., $\operatorname{Prob}(A \mid B)>\operatorname{Prob}(A)$ if and only if $\operatorname{Prob}(\mathrm{B} \mid \mathrm{A})>\operatorname{Prob}(\mathrm{B})]^{9}$

We can see how this reciprocity works most clearly in the case of the observational confirmation of a theoretical hypothesis: If a theory probabilistically supports the occurrence of a certain event, then the observation of the event confirms (raises the probability of) the theory. Hypothetico-deductive confirmation, where the hypothesis logically entails the occurrence of the event, is its limiting case. ${ }^{10}$

Some people may worry that RPS legitimizes the fallacy of affirming the consequent, i.e., RPS appears to imply that whenever A supports B ('If A, then B') and we can affirm the consequent, $\mathrm{B}$, we can thereby conclude that the antecedent, $\mathrm{A}$, holds because the supporting relation between A and B is mutual, according to RPS. This reasoning is clearly unacceptable. For example, the proposition that every member of the Beatles is dead supports the proposition that John Lennon is dead, and we can affirm that John Lennon is dead; but it is absurd to conclude on these grounds that every member of the Beatles is dead. This, however, is not what RPS implies. RPS only implies that affirming John Lennon's death raises the probability —only modestly in this case—-that every member of the Beatles is dead. The reasoning of affirming the consequent in this weaker sense is legitimate, and not a fallacy at all. ${ }^{11}$ 
The last set of preliminary remarks concerns the classification of epistemological theories. It is tempting to regard a theory of epistemic justification as coherentist if it allows coherence to justify beliefs. But such characterization is too simplisticjustification by coherence is not necessarily coherentist justification. An epistemologist may acknowledge that coherence of beliefs increases their justification only because coherence allows their existing justification to be channeled among them, just as logical entailment allows justification to be channeled from basic to derived beliefs in foundationalism. Such a theory should not be considered coherentist in my view. I suggest we take coherentism to be the view that coherence of beliefs generates justification for them. To see the advantage of this characterization, consider the regress argument for foundationalism. The gist of the argument is that a belief is justified by another belief only if the justifying belief is itself justified, and thus if any beliefs are to be justified at all without infinite regress or circularity, there must be basic beliefs that are justified independently of any other beliefs. A key assumption in this argument is that relations among beliefs only serve as channels of justification, and never generate justification. Coherentists challenge this 'pipeline' model of justification, asserting that relations among beliefs can be a source of justification on their own. ${ }^{12}$ Seen from this perspective, what distinguishes coherentism from other theories of epistemic justification is its claim that coherence generates justification for beliefs.

There are of course other ways of classifying epistemological theories, of which I want to mention two, so that they will not be confused with our classification by sources of justification. It is commonplace to characterize foundationalism by its two-tiered structure of basic and derived beliefs. The name 'foundationalism' appears particularly apt for the view that basic beliefs provide the foundation for derived beliefs. However, as some epistemologists have noted, coherentism has its own basic beliefs-viz., coherent beliefs, which are justified by their property of being coherent with one another, and not by justification channeled from outside the coherent system. In other words, coherentists are merely suggesting 'an unusual condition for proper basicality' (Plantinga [14, p. 79]). ${ }^{13}$ Once we see coherentism in this light, it is not difficult to conceive of a version of coherentism with a two-tiered structure - a version in which basic beliefs are justified directly by their coherence while derived beliefs are justified indirectly by inference from basic beliefs. ${ }^{14}$ Alvin Plantinga calls this version of coherentism 'an impure or mixed variety' (ibid.), but these terms are somewhat misleading. Presumably Plantinga calls it 'mixed' because it allows both direct justification of basic beliefs by coherence and indirect justification of derived beliefs by inference; but for that matter standard foundationalism is also 'mixed' since it allows both direct justification of basic beliefs and indirect justification of derived beliefs. A 'pure' version of foundationalism would only acknowledge direct justification of basic beliefs. The existence of a two-tiered structure is then conceptually independent of our classification of epistemological theories by sources of justification. Both foundationalism and coherentism can be with or without a two-tiered structure.

Another approach to classifying epistemological theories pays attention to the directions of justification. Susan Haack [8, p. 19] states that 'a theory which does not require one-directionality [of justification] cannot be foundationalist.' According to Haack, such a theory must be either a version of coherentism or a hybrid between foundationalism and coherentism, which she calls foundherentism. This classification 
relies on the way justification is channeled - either one-directionally or multidirectionally - while our classification relies on the way justification is generated. It is not surprising that some theories which allow multi-directional channeling of justification, and therefore 'cannot be foundationalist' in Haack's sense, turn out to be foundationalist in our analysis. Indeed what Haack calls foundherentism is a version of foundationalism in our classification since relations among beliefs do not generate any justification in it. ${ }^{15}$

\section{Increase in Existing Empirical Justification by Coherence}

This section examines cases of an increase in existing empirical justification of beliefs by their coherence. Cases of this kind are common. For example, when beliefs that are justified by different perceptual modalities, such as sight, sound, and smell, show a high degree of coherence, we trust them more. Similarly when beliefs based on testimonies of different witnesses, whom we already trust to some degree, fit together nicely, we become more confident of their truth. In such cases each belief has some degree of empirical justification independently of its coherence with other beliefs, and the role of coherence is to increase this existing empirical justification. I distinguish these cases from more controversial cases to be discussed in the next two sections where beliefs with no independent empirical justification become justified by their coherence.

Note here that the independent justification each belief has must be empirical, and not a priori. We often have good non-empirical reasons to assign different probabilities to various contingent propositions. For example, it is reasonable to assign a higher probability to the proposition that it will be raining somewhere in the world tomorrow than to the proposition that it will be raining in New York tomorrow, even if we have no information at all on tomorrow's weather. This is because the first proposition is less specific and therefore has more chances of being true. Such non-empirical reasons alone may be sufficient for accepting some contingent proposition. However, this is not the kind of independent justification we will be looking for. Our subject of investigation is empirical justification, and thus we will not be interested in an increase in non-empirical justification. This section only deals with cases of an increase in existing empirical justification by coherence, where each of the coherent beliefs is already more justified by empirical evidence than it is for non-empirical reasons alone.

Cases of an increase in existing empirical justification by coherence are so strong that denying it would considerably diminish the plausibility of any epistemological theory. As a result, many foundationalists-including the two most prominent in this century, C. I. Lewis and Roderick Chisholm-have co-opted the idea of an increase in existing justification by coherence. ${ }^{16}$ Thus, the issue is no longer highly controversial among epistemologists. It is, however, still worthwhile to investigate how this increase in justification occurs; in particular, whether coherence generates the additional justification. Chisholm [3, pp. 85-6], for example, states that his foundationalist account of knowledge: 
....includes mutual support among the sources [emphasis added] of epistemic justification. It would be a mistake, therefore, to say that foundational theories and coherence theories of epistemic justification must be incompatible.

In my view this is a needless concession on the part of Chisholm, and only obfuscates the issue. Closer examinations below reveal that cases of an increase in existing empirical justification by coherence, which prompted Chisholm to make his concession, only require that coherence channel existing empirical justification. Coherence (mutual support) is not the source of the additional justification in them.

Let us begin with a case of two coherent beliefs. Suppose I am watching a skating competition on TV and come to believe that the skater on screen is Chinese based on the visual perception of her facial features. Let us assume that this belief is justified. ${ }^{17}$ Let us also assume that I have appropriate background beliefs about different peoples and their languages, such that it is reasonable for me to make an inference from proposition (A) that the skater is Chinese, to proposition (B) that the skater speaks Chinese. Consequently, I am also justified in believing that the skater speaks Chinese. There is nothing unusual in this reasoning. The supporting relation between the two propositions serves as a channel of justification from (A) to (B). Recall, however, that by RPS any non-trivial probabilistic support is reciprocal. In other words, given the same background beliefs, the proposition (B) that the skater speaks Chinese supports in return the proposition (A) that the skater is Chinese. This does not mean that the two propositions are actually justified by each other. They are mutually supportive only in the hypothetical sense that if (A) is justified, then so is (B); and conversely if (B) is justified, then so is (A). In the present case, (A) has independent empirical justification, and thus the first of these two hypothetical supports - from (A) to (B) — becomes actual; but the support in the opposite direction — from (B) to (A) — remains hypothetical since (B) has no independent empirical justification.

The point here is that even when the actual justification is one-directional, the underlying supporting relation is still mutual, and thus justification may be channeled in the opposite direction. In other words, if it is (B) that gets independent empirical justification—for example, if I come to believe that the skater speaks Chinese on the basis of auditory information - then justification is channeled from (B) to (A). Of course, if neither (A) nor (B) has independent empirical justification, there is no actual channeling of justification in either direction. The most interesting case for our purpose is the one in which both (A) and (B) have independent empirical justification-for example, visual experience supports proposition (A) and auditory experience supports proposition (B). When this happens, both directions of hypothetical support are activated, and thus in addition to direct empirical justification, both beliefs receive indirect empirical justification via the other belief. The point to note is that even in this case of actual twodirectional support, the coherence relation only channels existing empirical justification whose justificatory source is sensory experience. Coherence does not generate the additional justification-it allows a transfer of justification.

Some people may worry that mutual justification would trigger a spiral of justification where (A) justifies (B), which in turn justifies (A), which in turn justifies (B) more, and so on indefinitely. This understandable concern arises from a wide-spread misconception about the transfer of justification. I want to draw attention, first, to the fact 
that the problem of spiraling justification is not unique to the case of two-directional justification; i.e., those who worry about a spiral of justification need to worry about it even in the case of one-directional justification. We already know that any probabilistic support is reciprocal although often only one-direction is activated by independent empirical justification. Consider again then the case where only proposition (A) receives independent justification, while proposition (B) receives no independent justification. Obviously, (A) justifies (B), and thus (B), which has no direct empirical justification of its own, receives indirect empirical justification. But then given the mutual hypothetical support between (A) and (B), it appears that (B) with its indirect justification now justifies (A) more, which in turn justifies (B) more, and so on indefinitely. Thus, onedirectional channeling of justification in which only one of the mutually supportive beliefs receives independent justification is no less problematic than two-directional channeling of justification, in which both beliefs receive independent justification.

Fortunately, there is no spiral of justification in either case. Let us begin with the case of one-directional channeling. It is not true, despite their mutual support, that when (B) receives empirical justification from (A), (B) in its turn justifies (A) more. The impression that it does arises from a confusion between two modes of transfer. In a transfer of goods - such as commodities, funds and energy - the original holder loses whatever is transferred while the new holder gains what the original holder loses. If the goods are transferred back, then the original holder regains what she lost. In the end, there is no gain from the initial state-we do not get rich by moving money back and forth between two accounts. The situation is different in a transfer of information in that the original holder does not lose the information transferred. For example, the teacher is no less knowledgeable after transferring information to her students. Both the original and new holders possess the information. Note, however, that the original holder gains nothing when the information is transferred back to her-the teacher does not gain any knowledge on the subject matter by reading her students' notes. Thus, in either model, there is no gain from the initial state when the same item is transferring back to the original holder.

Epistemologists are well aware that epistemic justification is transferred like information; i.e., when justification is transferred from belief (A) to belief (B) because (A) supports (B), (A) still retains its original justification. However, many epistemologists also speak of 'justificatory power' which strongly suggests that it is transferred like goods. If justification is transferred like information, the original belief retains its justification even after the justification is transferred; if justification is transferred like goods, the original belief gains justification when it is transferred back from another belief. By mixing the two models, we get the false impression that the original belief retains its justification and gains some more when justification is transferred back from another belief. However, once we recognize that justification is not transferred like goods and apply the information model consistently, there is no need to prohibit cyclical transfer of justification since cyclical transfer does not increase the amount of information (justification) in a spiral fashion.

The situation remains the same in the case of two-directional channeling where both of the mutually supportive beliefs have independent justification. The right analogy here is not an exchange of goods but an exchange of information. When two people with different pieces of information exchange them, each of them gets more information; but 
there is no further increase when they send the information back to the original holder. We cannot gain knowledge by sending the same old email back and forth. Similarly, when beliefs (A) and (B) are coherent and each of them has independent empirical justification, they receive additional justification from the other belief. Thus, beliefs get more justified because of their coherence, but there is no spiral of justification in which the same justification is used over and over in support of the same belief.

The point also applies to cases involving three or more beliefs. Suppose a set of three or more beliefs is coherent in the sense that each member of the set is supported by the remainder of the set. In other words, each member becomes justified if the remainder of the set has independent empirical justification. No direction of this mutual support may be activated in the absence of independent empirical justification, or perhaps only some of them may be activated if there is independent empirical justification for only some of the coherent beliefs. It is when each member has independent empirical justification that all directions of mutual support become activated for mutual justification. This is because each belief, which is already empirically justified independently, receives additional justification from the remainder of the set. Since there would be no such increase in justification if the beliefs were not coherent, it is fair to say that coherence increases existing empirical justification for beliefs; but coherence only serves as a channel of justification, and is not a source of justification. Thus, regardless of the number of beliefs involved, the increase in existing empirical justification by coherence does not require coherence to generate additional justification. The role of coherence is to provide a channel of justification from one belief(s) to another, as relations among beliefs have always been assumed to do in non-coherentist theories of justification. Thus, non-coherentists can accommodate an increase in existing empirical justification by coherence without conceding any point to coherentists.

\section{Justification By Coherence From Scratch}

This section examines justification by coherence where unlike those in the preceding section none of the coherent beliefs has independent empirical justification. The phrase 'justification by coherence' in the following discussion refers to this type of justification by coherence from scratch. Here are some examples. I hear testimonies of different witnesses, whom I have no reason to trust, but their testimonies show a high degree of coherence-e.g., the details of their stories match closely and complement each other. It appears that such mutual support lends at least some credibility to the testimonies, and I may come to believe them as a result. When this happens, my belief in each testimony has no independent justification but my beliefs are nonetheless justified to some degree, it seems, because of their coherence. Similarly, even when I have no trust in my individual perceptual beliefs_-for example, after taking a drug known to cause perceptual delusions - a high degree of coherence among my perceptual beliefs still seems to make them somewhat credible. Coherence apparently generates justification in these cases. For, the beliefs involved have no independent empirical justification that can be channeled through their mutual support, and thus we cannot explain these cases in the way we did in the preceding section by the channeling of justification among the beliefs. 
We must admit that the intuitive appeal of justification by coherence from scratch is considerably weaker than that of an increase in existing justification by coherence. It is hard to find cases where the beliefs involved have clearly no independent empirical justification but still become justified by their coherence. In the case of coherent testimonies, for example, it is arguable that we bestow some amount of credibility to any testimony even if it is given by a total stranger, or even by a known liar, because even liars typically tell more truths than falsities in their life time. In other words, whenever we hear a testimony, we may automatically assign a higher probability than its propositional content alone warrants. If that is the case, then each belief we form already has some independent empirical justification, and thus we can apply the analysis provided in the preceding section that explains an increase in existing justification. The default assignment of credibility is even more likely in the case of perceptual beliefs; i.e., it seems we consider any perceptual belief credible in the absence of some evidence to the contrary. Mere undercutting defeaters, such as the memory of taking a hallucinatory drug, may not be enough to eliminate the default credibility of perceptual beliefs entirely. ${ }^{18}$ It may even be argued that any belief is more credible than its propositional content alone warrants simply by the fact that it is believed. If that is correct, then all cases of justification by coherence may be cases of an increase in existing justification, where coherence channels existing justification among the beliefs.

I believe however that we can circumvent this difficulty by introducing empirical evidence that each of the coherent beliefs is false, so that we can neutralize their default credibility. Suppose, for example, we hear testimonies from known liars, each of whom is interested in tarnishing the person they are testifying about to such an extent that we would give less credibility to what each of them says than their propositional contents warrant. It appears nonetheless that a surprising degree of coherence in the details of their testimonies, which I assume are given independently, would make them credible at least to some extent. Beliefs based on these testimonies are then justified to some extent because of their coherence though each of them has no independent justification. This kind of thought experiment is not decisive. We may be simply mistaken in our intuitive judgment, especially with contrived cases like this. However, the use of contrary evidence seems to enable us to construct at least prima facie cases of justification by coherence where each of the coherent belief carries no default credibility. I will analyse these apparent cases of justification by coherence-assuming tentatively that they are genuine - to understand how it might be possible. In this section I will describe what I consider the most promising account of justification by coherence from scratch, and then in the next section I will examine, relying on this account, whether coherence generates justification in these cases.

I want to start with cases in which beliefs remain unjustified no matter how coherent they are, so that we will have a better understanding of conditions required for justification by coherence. Here is one such case. I come to believe on some empirical grounds that the skater on screen is Chinese and make an inference from this- together with background beliefs - that she speaks Chinese, lives in China, and likes Chinese food. Suppose, however, I realize that the initial empirical evidence that she is Chinese was faulty. As a result, I am left with a highly coherent collection of beliefs none of which has independent empirical justification. It is quite clear in this case that these beliefs have no credibility despite their coherence. This is one version of the so-called 
isolation problem of coherentism, in which a coherent set of beliefs has no contact with reality, and thus should not be justified despite their coherence. My purpose in presenting this case is not to dispute justification by coherence. The isolation problem reveals that coherentism without appropriate constraints would justify many beliefs that should not be justified. The obvious response to this challenge of too-much-justification is to introduce appropriate constraints, which should help us understand the mechanism of justification by coherence.

The skater case above points to an important feature that affects the success of justification by coherence; namely, the way the coherent beliefs are formed. We find a case of justification by coherence compelling when each of the coherent beliefs is formed independently. ${ }^{19}$ This is because surprising coherence of independently formed beliefs requires an explanation, and one natural explanation is the reliability of the beliefforming mechanism responsible for these beliefs. If the belief forming mechanism is reliable, then the beliefs are more likely to be true than their propositional contents alone indicate; consequently, these beliefs are empirically justified because of their coherence (see BonJour [1, Sec. 8.3]). Thus, an apparent connection exists between the coherence of beliefs and their justification provided the coherent beliefs are formed independently of each other. On the other hand, in clearly unsuccessful cases of justification from which the isolation objection arises, there is no such independence among the beliefs in question. For example, in the skater case just described, all beliefs, except for the first one, are formed inferentially on the basis of other beliefs. This lack of independence in belief formation eliminates the need for explaining coherence by the reliability of the belief-forming mechanism. For, we have an obvious and trivial explanation of coherence-namely, inferred beliefs are coherent with beliefs from which the inference is made. Similarly, it is not surprising that non-independent testimonies given by a single witness - or a group of conspiring witnesses - are coherent. Their wishes to make their story convincing explain the coherence; for, they would take inferential relations into consideration in adjusting their testimonies to fit those of others. Consequently, we have no reason to postulate the reliability of the belief-forming mechanism in order to explain coherence of our beliefs if they are based on non-independent testimonies.

Here is then the picture of justification by coherence that emerges from the consideration of the isolation problem. We can justify beliefs by their coherence provided there is no trivial explanation of their coherence. According to this account, we must set aside inferentially formed beliefs when we try to justify beliefs by their coherence because inferentially formed beliefs are coherent for obvious reason. Of course, inference is relevant to justification, but it is relevant only derivatively when we already have noninferential 'basic' beliefs justified by their coherence, and when we can derive further beliefs by inference from them. The resulting theory is what Plantinga [14, p. 79] calls a 'mixed' version of coherentism in which there is a distinction between basic and derived beliefs.

I believe this version of coherentism can handle the isolation problem well, but it still faces a variety of other objections, of which I will discuss what I consider the two most important. They are (1) the existence of alternative accounts of coherence, and (2) the requirement of justified meta-beliefs. The first objection is that even when beliefs are formed non-inferentially and there is no trivial explanation of coherence, there are still many alternative accounts, such as Berkelean phenomenalism with divine coordination, 
which can also explain the coherence of beliefs; and these accounts make the explanation of coherence by the reliability of the belief-forming mechanism unnecessary. The second objection concerns the necessity of the justified meta-belief that our beliefs are coherent. I will deal with the problem of the meta-belief in the next section. The remainder of this section addresses the problem of alternative accounts of coherence.

I have stated that the goal of this section is to provide the most promising account of justification by coherence. However, I am not going to dispute or discredit alternative accounts of coherence in order to defend my account. I want to argue instead that coherence confirms the reliability of the belief-forming mechanism as long as the reliability hypothesis explains their coherence, even if alternative hypotheses can also explain the coherence. The key in this argument is the Reciprocity of Probabilistic Support (RPS) explained in Section I. According to this principle, any non-trivial probabilistic support is reciprocal; and thus, as long as the hypothesis that our beliefforming mechanism is reliable makes it more likely that our beliefs are coherent, the fact that our beliefs are indeed coherent confirms (i.e., raises the probability of) the reliability hypothesis. Obviously, RPS applies to alternative hypotheses as well. If an alternative hypothesis makes it more likely that our beliefs are coherent, the fact that our beliefs are indeed coherent confirms it as well. But that does not matter. Recall that we have decided to understand justification probabilistically without setting any threshold of justification at which a belief changes its status from being unjustified to being justified. The problem we are interested in here is thus whether coherence makes it more likely that the beliefforming mechanism is reliable. It is irrelevant to this question that coherence also makes it more likely that some other hypothesis is correct. Of course, if we have to cross some threshold of credibility at which the justificatory status of our belief changes, then it is not enough to show that coherence confirms (raises the probability of) the reliability hypothesis. We would have to dispute or discredit alternative accounts of coherence, so that the reliability hypothesis will receive a sufficiently high probability. But our modest goal is to show that coherence of non-inferentially formed beliefs makes it more likely than otherwise that our belief-forming mechanism is reliable. If the reliability of the belief-forming mechanism is one of the hypotheses that explain (and thus raise the likelihood of) the coherence of our beliefs, then by RPS the fact that our beliefs are indeed coherent confirms (raises the probability of) the reliability hypothesis along with other hypotheses that also explain the coherence.

Unfortunately this is not the end of the story. There is one thorny problem that still threatens the argument. A reliable belief-forming mechanism is likely to generate true beliefs; consequently, if the coherence of beliefs makes it more likely that the beliefforming mechanism is reliable, then other things being equal coherence should make our beliefs more likely to be true. But other things may not be equal. I have granted that coherence confirms not only the reliability hypothesis but certain other hypotheses as well, and according to some of them, coherent beliefs may be less likely to be true. If that is the case, and if these twisted hypotheses are sufficiently credible, then the overall impact of coherence on the beliefs' truth is unclear even if coherence confirms the reliability hypothesis.

What worries me here is not the Cartesian demon hypothesis. For, coherent beliefs are no less (and no more) likely to be true than incoherent beliefs according to the standard demon hypothesis. Other well-known sceptical hypotheses, such as the brain-in- 
a-vat story, are also neutral with respect to the impact of coherence on truth; i.e., coherent beliefs are no less (and no more) likely to be true according to them. However, we can think of a cynical deity who deliberately puts into our minds false beliefs that are coherent, in which case coherence is a sign of falsity. Of course, some accounts of coherence, other than the reliability hypothesis, make it more likely that the coherent beliefs are true. For example, the cynical deity's benevolent counterpart may put into our minds true beliefs that are coherent, in which case coherence is a sign of truth. If the positive and negative impacts of these alternative hypotheses off-set each other, then other things are equal after all; and thus the reliability hypothesis, whose likelihood is raised by the coherence of beliefs, will raise the likelihood that the beliefs in question are true. But it is hard to assess the total impact of all possible explanations of coherence. Thus, there remains the possibility that given all possible explanations of coherence, the coherent beliefs are no more likely to be true.

In the next section I assume, for the argument's sake and in favor of coherentism, that the positive and negative impacts of the alternative accounts of coherence off-set each other, and thus coherent beliefs are more likely to be true; and investigate whether coherence generates justification when beliefs are justified by coherence.

\section{Does Coherence Generate Justification?}

This final section argues that justification by coherence as explained in the preceding section results from the channeling of justification, and thus coherence need not generate any justification. This may sound strange because unlike cases in Section II where each of the coherent beliefs already has some independent empirical justification, we are here dealing with coherent beliefs with no such independent justification to channel. Channeling of empirical justification among coherent beliefs is therefore out of the question. However, justification may be channeled from outside because of their coherence. In that case it is fair to say that coherence justifies beliefs even if it generates no justification. More specifically I will argue that the justification in question is channeled from the meta-beliefs about the coherent beliefs.

It has been one of the major criticisms of coherentism that justification by coherence requires the meta-belief that the beliefs in question are indeed coherent, and consequently even coherentism faces an infinite regress in justifying increasingly higher levels of meta-beliefs unless some non-coherentist justification of meta-beliefs is available. Holistic coherentism is often thought to be a solution to the regress problem, but it cannot stop the regress of higher-order justifications since holistic justification of various levels of meta-beliefs would require a still higher level of justified meta-belief that these meta-beliefs are coherent. The issue of meta-beliefs is a formidable challenge for coherentism, and it forced a leading coherentist to introduce the presumption that the epistemic agent's beliefs about her own belief system is largely true (BonJour [1, pp. 101-6]). This move is highly controversial since it makes coherentism vulnerable to the same kind of objections that coherentists customarily raise against foundationalism in order to motivate coherentism; it is difficult to defend the presumption that a certain type of beliefs is largely true without further justification, while disputing the notion of basic 
beliefs in foundationalism (Brueckner [2]). Some critics even consider this presumption foundationalist (Pollock [16, p. 73n]). It is premature, however, to write off coherentism even if the presumption is foundationalist; for, the necessity of meta-beliefs with noncoherentist justification does not by itself establish that coherence generates no justification. We need to address two further questions before we draw this conclusion. First, there is the possibility that the meta-belief may be justified non-empirically, in which case coherence still needs to generate the empirical part of justification. Second, even if the meta-belief must be justified empirically, that justification may never get channeled to the object beliefs that are coherent, in which case coherence still needs to generate the justification for the object beliefs.

To see the significance of these two questions, I want to make a comparison between the justification of coherent beliefs with the justification of logical truths. Suppose I come to believe that snow is either white or not white, and I am confident that I can justify this belief solely by its propositional content independently of any empirical evidence since it is logically true. It appears we have a case of purely a priori justification, but on second thought the justification of this belief seems to require the justification of the meta-belief that the belief in question is indeed logically true. Interestingly, however, in the case of logical truth, epistemologists are reluctant to conclude — not for this reason any way — that our belief in logical truth is justified in part on empirical grounds. This is presumably because of the following two reasons - the required meta-belief may not have to be justified empirically, or even if the meta-belief must be justified empirically, this justification may never get channeled to the object belief in logical truth.

Coherentists may make similar points with respect to justification by coherence; namely, even if the justification of beliefs by their coherence requires the justified metabelief that the beliefs in question are indeed coherent, the required justification may not be empirical; and further even if the justification is empirical, it may never get channeled to the object beliefs. In either case, we would be unable to explain away cases of justification by coherence as resulting from channeling of empirical justification from meta-beliefs. Change in their justificatory status would have to be explained in some other way - possibly by the generation of justification by coherence. In this section I wish to establish that coherence need not generate justification by showing that justification by coherence does require empirically justified meta-beliefs, and further that their empirical justification does get channeled to the object beliefs that are coherent.

Let us take a closer look at the meta-belief - that we have coherent beliefs - that is required for justification by coherence. It is helpful to separate two components in it. First, there is the belief that we have beliefs with certain propositional contents; and second there is the belief that these propositions are coherent. As to the second component, coherentists can plausibly maintain —and I grant this in their favor-that the judgment of coherence is a priori. As to the first component, however, we cannot plausibly maintain that the meta-beliefs about the contents of our beliefs are justified a priori. The discovery that we have beliefs with certain propositional contents is surely empirical - e.g., I cannot tell whether I believe today is Tuesday or Wednesday on purely a priori grounds. We must get some information about the state of our mind. Thus, the central problem about the meta-belief in coherentism is that justification by coherence requires non-coherentist justification of meta-beliefs about the propositional contents of 
our beliefs. We cannot plausibly maintain that these meta-beliefs are in turn justified by their own coherence because such coherentist meta-justification would in turn require a justified meta-meta-belief about the meta-beliefs' coherence, and we will soon fall into an infinite regress of higher-order justifications. Any attempt to stop the regress by holistic coherentism is frustrated because that would require an already justified metabelief that the beliefs in question are coherent.

The separation of the two components-the belief about the propositional contents of our beliefs, and the belief in the coherence of the propositions in questionhelps us narrow down our focus; and it appears that justification by coherence does require empirically justified meta-beliefs for the first component. This raises the question: Does this point also apply to the belief in logical truth? In other words, can we separate two components in the meta-belief that the belief we have is logically true, and conclude that one of the two components requires empirical justification? The two components should be: (1) the belief that we have a belief with a certain propositional content, and (2) the belief that the proposition in question is logically true. It is clear that the first belief about the content of our belief is empirical. Note, however, that we need this component only if we want to justify the belief we have. It is unnecessary if we only want to justify the proposition itself. Suppose, for example, I believe for no good reason that I believe the number of the stars in the universe is either even or not even. We may assume that I have actually no belief about the number of the stars in the universe. My meta-belief is then unjustified and false. Suppose, now, that I believe on a priori grounds in the logical truth of the proposition that the number of the stars in the universe is either even or not even. Under these conditions, I have a priori justification for this proposition though I have no justification for the belief I have. The latter requires the empirically justified meta-belief that I do believe the number of the stars in the universe is either even or not even. Thus, as far the justification of logically true proposition is concerned, there is no need for any empirically justified meta-belief.

In contrast to this, we cannot justify beliefs by coherence without the empirically justified meta-beliefs about the contents of our beliefs even for the purpose of justifying the believed propositions. Recall that the ground for justification by coherence, according to the analysis provided in the preceding section, is that coherence of non-inferentially formed beliefs demands an explanation, and that one natural explanation is the reliability of the belief-forming mechanism responsible for the beliefs. It is crucial in this route to justification that the coherent propositions are the contents of our actual beliefs-and further that these beliefs are formed non-inferentially. Otherwise, there is no need for explanation by the reliability hypothesis. In this respect, justification by coherence is quite different from justification of logical truth. In the case of logical truth, we can set aside the issue of whether we actually believe it or not, and focus solely on the proposition for its justification. In the case of justification by coherence, on the other hand, if we set aside the issue of whether we actually have these beliefs or not, and focus solely on propositions for their justification, then we end up accepting the most coherent system of propositions we can think of, which has no connection to actual beliefs we form with cognitive spontaneity, and which is therefore totally isolated from reality.

We have just discovered that justification by coherence, unlike justification of logical truth, requires the empirically justified meta-beliefs about the contents of our actual beliefs. The final question is whether this empirical justification of the meta- 
beliefs is channeled to the object beliefs that are coherent. Again the account of justification by coherence developed in the preceding section provides an answer. Noninferentially formed beliefs are justified by their coherence because the hypothesis that the belief-forming mechanism responsible for them is reliable explains the coherence. The basis of this reasoning is RPS. The reliability hypothesis makes it more likely that our non-inferentially formed beliefs are coherent, and thus by RPS the coherence of our non-inferentially formed beliefs in turn makes it more likely that our belief-forming mechanism is reliable. In other words, the reliability hypothesis and the coherence of non-inferentially formed beliefs are mutually supportive. Consequently, when one of them receives independent empirical justification, it is channeled to the other. This is why the reliability hypothesis becomes justified when we obtain justification for the meta-belief that our non-inferentially formed beliefs are coherent. This justification for the reliability hypothesis is channeled further to the coherent object beliefs since beliefs produced by a reliable belief-forming mechanism are likely to be true. In this way empirical justification is channeled from meta-beliefs to object beliefs, and it is coherence that licenses the channeling. To conclude, beliefs with no independent empirical justification can be justified by their coherence, but even in such cases coherence need not generate justification for the beliefs.

\section{Conclusion}

We identified two types of cases where coherence plays a positive role in epistemic justification. First, in some cases coherence among beliefs increases their existing empirical justification. The role of coherence in these cases is simply to serve as channels of justification among the beliefs; coherence need not generate any empirical justification. We also examined cases of justification by coherence from scratch, where none of the coherent beliefs has independent empirical justification. We discovered here as well that coherence need not generate empirical justification; the role of coherence is to license the channeling of empirical justification from meta-beliefs to object beliefs. Thus, coherence either serves as a channel of justification or licenses the channeling of justification without generating any empirical justification on its own.

This diagnosis is based on our analysis of the way beliefs can be justified by their coherence; and there remains the possibility that beliefs may be justified in some surprising way by their coherence, in which coherence generates empirical justification. For a complete repudiation of coherentism-i.e., to establish that all justification by coherence results from channeling - we need to eliminate this possibility, but that is not our objective in this paper. Our modest goal is to analyse apparent cases of justification by coherence that make coherentism appealing, and present a non-coherentist account of them. We discovered that beliefs can indeed be justified by their coherence in these cases, which explains the strong appeal of coherentism; however, we also discovered that the role of coherence in them does not support coherentism in epistemology, which in our classification is the view that coherence among beliefs can generate empirical justification. 
As mentioned at the outset, philosophers take coherentism seriously for positive and negative reasons. The positive reason is apparent cases of justification by coherence, but our analysis revealed that coherence need not generate justification in them. We have therefore no positive reason to endorse coherentism. Coherentists are thus left with only negative arguments against alternative theories of epistemic justification, but in the absence of positive reason, failure of its alternatives seems to support scepticism more strongly than it does coherentism. ${ }^{20}$

Rhode Island College

\section{REFERENCES}

1. BonJour, L., The Structure of Empirical Justification (Cambridge, MA: Harvard University Press, 1985).

2. Brueckner, A., 'Problems with internalist coherentism', Philosophical Studies 54 (1988), pp. 53-60.

3. Chisholm, R., Theory of Knowledge, 3d ed. (Englewood Cliffs: Prentice-Hall, 1989).

4. Fritzman, J. M., 'Against coherence', American Philosophical Quarterly 29 (1992), pp. 183-91.

5. Firth, R., 'Epistemic merit, intrinsic and instrumental' in J. Troyer (ed.), In Defense of Radical Empiricism: Essays and Lectures by Roderick Firth (Lanham, MD:

Rowman and Littlefield, 1998), pp. 259-71. Originally appeared in Proceedings and Addresses of the American Philosophical Association 55 (1981).

6. Goldman, A., Epistemology and Cognition (Cambridge, MA: Harvard University Press, 1986).

7. Goldman, A., 'Internalism exposed', Journal of Philosophy 96 (1999), pp. 271-93.

8. Haack, S., Evidence and Inquiry: Towards Reconstruction in Epistemology (Oxford: Blackwell, 1993).

9. Kahneman, D., P. Slovic and A. Tversky (eds.), Judgment under Uncertainty: Heuristics and Biases (Cambridge: Cambridge University Press, 1982).

10. Kornblith, H., 'The unattainability of coherence', in J. Bender (ed.), The Current State of The Coherence Theory (Dordrecht: Kluwer, 1989), pp. 207-14.

11. Kvanvig, J., 'In defense of coherentism', Journal of Philosophical Research 22 (1997), pp. 299-306.

12. Lewis, C.I., An Analysis of Knowledge and Valuation (La Salle: Open Court, 1946).

13. Olsson, E., 'Coherence and the modularity of mind', Australasian Journal of Philosophy 75 (1997), pp. 404-11.

14. Plantinga, A., Warrant: The Current Debate (Oxford: Oxford University Press, 1993).

15. Pollock, J., Knowledge and Justification (Princeton: Princeton University Press, 1974).

16. Pollock, J., Contemporary Theories of Knowledge (Savage, MD: Rowman and Littlefield, 1986).

17. Sosa, E., 'The raft and the pyramid: coherence versus foundations in the theory of knowledge' in his Knowledge in Perspective (Cambridge, UK: Cambridge University 
Press, 1991), pp. 165-91. Originally appeared in Midwest Studies in Philosophy 5 (1980).

${ }^{1}$ See Pollock [15, pp. 27-8] and Sosa [17, pp. 18-20]) among others for clear presentations of the isolation problem of coherentism. See Pollock [16, p. 81] on the problem of basing in coherentism, and Kvanvig [11] for a response from a coherentist perspective. See Kornblith [10] on the problem of feasibility in coherentism, and Olsson [13] for a coherentist's attempt to circumvent the difficulty.

${ }^{2}$ The desirable consequence that provides pragmatic justification may be an increase in true beliefs. For example, Roderick Firth [5, p. 260] discusses a case of a researcher whose belief in his recovery from his illness against evidence has instrumental epistemic merit in that the belief encourages him to continue working and contribute to the growth of knowledge. Though the merit is epistemic, justification is still pragmatic.

${ }^{3}$ Cf. Alvin Goldman's [6, pp. 25-6] distinction between regulative and nonregulative systems of epistemic norms.

${ }^{4}$ In his recent challenge to internalism Alvin Goldman [7, p. 278] states: 'Strong internalism threatens a drastic diminution in the stock of beliefs ordinarily deemed justified [my emphasis]... This is a major count against this type of theory.' In my view if a theory with a clear objective - such as a theory in agent-centered teleological epistemology, or a theory in quantum physics for that matter - has consequences that are in conflict with ordinary judgment, then it is a major count against relying on ordinary judgment in our theorizing.

${ }^{5}$ Note that we are still interested in justification of beliefs, as opposed to justification of propositions; it becomes crucial later in our discussion that the agent actually believes propositions to be justified. The point of the present remark is not to set aside doxastic justification and focus on propositional justification, but to set aside the issue of basing and focus on justifiability of beliefs. Thanks to two anonymous referees for helping me set this straight.

${ }^{6}$ In other words, people often hold set $\left\{\mathrm{B}_{1}, \ldots, \mathrm{B}_{\mathrm{N}}\right\}$ of beliefs such that for each $\mathrm{Bi}(1 \leq \mathrm{i}$ $\leq \mathrm{N}), \operatorname{Prob}\left(\mathrm{B}_{\mathrm{i}} \mid \mathrm{B}_{1} \& \ldots \& \mathrm{~B}_{\mathrm{i}-1} \& \mathrm{~B}_{\mathrm{i}+1} \& \ldots \& \mathrm{~B}_{\mathrm{N}}\right)>\operatorname{Prob}\left(\mathrm{B}_{\mathrm{i}}\right)$. This is the kind of mutual support C. I. Lewis [12, p. 338] calls 'congruence'.

${ }^{7}$ The qualification 'non-trivial' excludes cases in which one of the two propositions has no chance of being true.

${ }^{8}$ We assume-in favor of coherentism-that beliefs in theorems of the probability calculus, with which we can prove the ubiquity of coherence, can be justified a priori.

${ }^{9}$ To verify this theorem, replace $\operatorname{Prob}(\mathrm{X} \mid \mathrm{Y})$ by its definiens $\operatorname{Prob}(\mathrm{X} \& \mathrm{Y}) / \operatorname{Prob}(\mathrm{Y})$ and use elementary algebra.

${ }^{10}$ RPS holds for logical entailment as well in the sense that if proposition A logically entails proposition $\mathrm{B}$ in a non-trivial way (i.e., $\mathrm{B}$ is not a logical truth), then $\mathrm{B}$ in return probabilistically supports $A$. For, if A logically entails $B$ while $\operatorname{Prob}(B) \neq 1$, then $\operatorname{Prob}(\mathrm{B} \mid \mathrm{A})=1>\operatorname{Prob}(\mathrm{B})$, and therefore $\operatorname{Prob}(\mathrm{A} \mid \mathrm{B})>\operatorname{Prob}(\mathrm{A})$ by RPS.

${ }^{11}$ This probabilistic legitimacy may explain the intuitive appeal of affirming the consequent. 
${ }^{12}$ More precisely it is the holistic version of coherentism that challenges the pipeline model. I will not discuss the linear version of coherentism, which accepts the pipeline model of justification, but which still resists foundationalism, since I know of no cogent defence of it.

${ }^{13}$ Similar points have been made by Sosa [17, pp. 178-81] and Fritzman [4, p. 184].

${ }^{14}$ It will be then only cognitively spontaneous (non-inferential) beliefs that are justified directly by their coherence. We will return to this version of coherentism later in Section III.

${ }^{15}$ Haack [8, p. 213] explicitly states that: 'According to the foundherentist criteria, the ultimate evidence with respect to empirical beliefs is experiential evidence, sensory and introspective.' See also figure 1.3 [8, p. 24], which indicates the foundationalist nature (in our sense) of her theory.

${ }^{16}$ See Lewis [12, Chap. 11] on 'congruence' and Chisholm [3, Chap. 7] on 'concurrence'.

${ }^{17}$ We need not assume that this is a basic belief. Our objective in this section is to analyse how beliefs that are somehow empirically justified independently of each other become more justified by their coherence.

${ }^{18}$ See Pollock [16, pp. 38-9] for the distinction between rebutting and undercutting defeaters.

${ }^{19}$ Independence of belief-formation does not entail—nor is it entailed by-independence of justification. Even if we form a belief independently of any other beliefs, it may still receive justification from other beliefs. On the other hand, an inferentially formed belief may have justification independently of the beliefs from which the inference was made. ${ }^{20}$ I would like to thank Daniel Flage, Chang-Seong Hong, Sheri Smith, anonymous referees and an associate editor of this journal for their valuable comments on earlier versions of this paper. 